\title{
Nonlinear Calibration of Thermocouple Sensor Based on Least Squares Support Vector Regression
}

\author{
Shengbo Zhang, Qingling Dai \\ Jiujiang University Jiujiang, Jiangxi, 332005, China \\ E-mail: B761127@tom.com
}

Keywords : nonlinear calibration, least squares support vector regression, sensor, power series

\begin{abstract}
Calibration is an important operation in the instrumentation industry for determining the relationship between the output(s) (or response) of a measuring instrument and the value of the input(s). This paper proposes a nonlinear calibration method based on least squares support vector regression (LS-SVR) with the output voltage of thermocouple sensor as input and the measured temperature output to eliminate the nonlinear errors in detection process. Firstly, the nonlinear calibrator, expressed by power series, was established based on the principle of inverse model. And then the parameters of the calibrator were identified by LS-SVR. Through this calibrator, the desired linear characteristics of thermocouple sensor could be obtained. Finally, platinum-rhodium 30- platinum-rhodium 6thermocouple (B-type) was taken as an example, and experimental results show that the proposed calibration method is efficient in the temperature range from $400^{\circ} \mathrm{C}$ to $1800^{\circ} \mathrm{C}$. And the method has an advantage of analytical expression of the calibration model.
\end{abstract}

\section{Introduction}

Sensors are important tools for information collection, widely used in all walks of life. There are a lot of nonlinear calibration methods of sensors [1]. On the whole, these are divided into hardware and software technology. Hardware calibration uses electronic circuits to correct the nonlinear error. Due to technique and electronic devices drift, so its applications are limited. In recent years, with development of computer technology, a variety of data processing software method came into being. Using artificial neural network (ANN) for nonlinear calibration of sensors is interesting $[2,3]$.

However, these methods have some shortcomings, which mainly reflected in the follows. (1) Local extremum and over learning problems: which maybe affect the generalization ability of ANN, therefore these methods are deeply dependent on the quantity and quality of the training samples; (2) Randomness: the learning results of ANN are related to initial value and the samples sequence, so the calibration is not unique; (3) Non-analyticity: ANN methods can not give out the mathematical analytic expression of the calibration model.

Support vector regression (SVR) bases on the structural risk minimization (SRM) rule, and Its topology structure is decided by support vector, which overcomes the shortcoming that ANN structure relies on the experience of designer [4]. Least square SVR (LS-SVR) is developed from standard SVR [5]. It substitutes equality constrains for inequality constrains of SVR, that is to say, it substitutes solving linear system of equations for quadratic programming. Thus, it reduces calculation complexity and speeds up solving.

In this paper, we propose a different method, which circumvents the problems associated with existing methods. The nonlinear calibration model is firstly expressed as power series polynomial form. And then LS-SVR is used to identify the parameters of calibration model. The platinum-rhodium 30platinum-rhodium 6-thermocouple sensor is finally taken as an example to verify these advantages.

\section{The principle of nonlinear calibration}

The measurement process with an intelligent sensor in modern instruments is commonly described in reconstruction categories [6]. Generally, it means that the measuring chain is divided into two elements, as shown in Figure 1. 


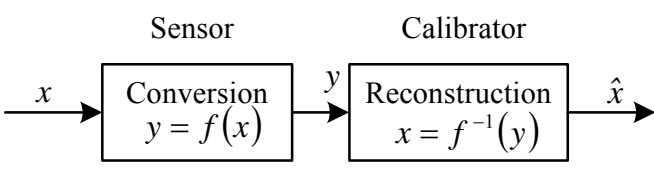

Fig.1 Measuring chain of intelligent sensor

The first element converts the input signal $x$ of the sensor to the output digital signal $y$. The role of the second element is to calculate the estimates $\hat{x}$ of the input signal $x$ based on the samples of the output signal $y$ in such a way that the estimates $\hat{x}$ are equal to suitable instantaneous values of $x$ [7].

The whole measurement process with the sensor can mathematically be described as follows: Let us assume that the conversion is described as $f(\cdot)$, so that we have

$$
y=f(x)
$$

The reconstruction consists of solving inverse model $f^{-1}()$ according to

$$
\hat{x}=f^{-1}(y) .
$$

Therefore, from (1) and (2), one obtains

$$
\hat{x}=f^{-1}(f(x))=x,
$$

which means that the measurement chain with reconstruction, as a whole, realizes an ideal measurement transducer.

\section{Nonlinear model of sensor}

In fact, the nonlinear relationship of thermocouple sensor can be denoted with the indexing table in the whole measuring range. Take the platinum-rhodium 30- platinum-rhodium 6 thermocouple (Btype) for example, the relationships between the measured temperatures (MT) and the output electromotive force (EMF), in the range of $0 \sim 1820^{\circ} \mathrm{C}$ The characteristics of a thermocouple sensor are hyperbolic in nature [8]. It may be mathematically represented with sufficient accuracy by the $\mathrm{N}$ terms of the power series as

$$
x=\omega_{1} y+\omega_{2} y^{2}+\ldots+\omega_{N} y^{N}+b=\sum_{i=1}^{N} \omega_{i} y^{i}+b
$$

where $y$ and $x$ represent the normalized measured temperatures and the output electromotive force, respectively. The parameter $b$ is the offset, and the coefficients $\omega_{i}, i=0,1, \ldots, N$, are constants of the thermocouple sensor.

In the case of direct modeling these coefficients are estimated using a LS-SVR. Using these values the response of the sensor may be computed as

$$
\hat{x}=\hat{\omega}_{1} y+\hat{\omega}_{2} y^{2}+\ldots+\hat{\omega}_{N} y^{N}+\hat{b} \text {. }
$$

where $\hat{\omega}_{i}, i=0,1, \ldots, N$, represent the estimated values of the coefficients.

To express it conveniently, formula (5) can be described in vector format as

$$
\hat{x}=\hat{\boldsymbol{\omega}}^{T} \mathbf{E}+\hat{b},
$$

where $\hat{\boldsymbol{\omega}}=\left[\hat{\omega}_{1}, \hat{\omega}_{2}, \ldots, \hat{\omega}_{N}\right]$ is the coefficient vector, $\mathbf{E}=\left[y, y^{2}, \ldots, y^{N}\right]$ is the input vector of samples.

For the thermocouple sensor, when the temperature $x=0$, the output EMF $y=0$. Therefore, the nonlinear calibration model should also be a zero-crossing, that means, we can simplify the model above as follows 


$$
\hat{x}=\hat{\boldsymbol{\omega}}^{T} \mathbf{E} .
$$

From the above analysis, we can conclude that the key to calibrate the sensor is to identify the model parameters $\hat{\boldsymbol{\omega}}$. Therefore, the nonlinear calibration model can be established by system identification method, and the process generally involves the following steps.

(1) Get the corresponding relationship between measured temperatures and the output electromotive force through the indexing table or the actual tests.

(2) Compose the training sample set $\left\{\mathbf{E}_{i}, x_{i}\right\}_{i=1}^{M} \in R^{N} \times R$ of the calibrator with the form of equation (6).

(3) Determine the model parameters $\hat{\boldsymbol{\omega}}$ of the calibrator by identifying the training samples.

(4) Compensate the actual output of thermocouple sensor, and record the calibration results $\hat{x}_{i}$.

(5) Compare the compensation results $\hat{x}_{i}$ with the measured temperatures $x_{i}$ to verify the effectiveness of the calibration process.

The calibration process of the nonlinear calibration for a thermocouple sensor based on system identification using LS-SVR algorithm is shown in Figure 2.

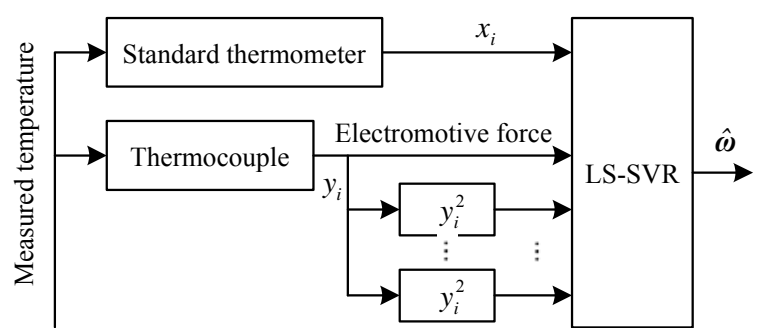

( a ) Identification scheme of calibration model

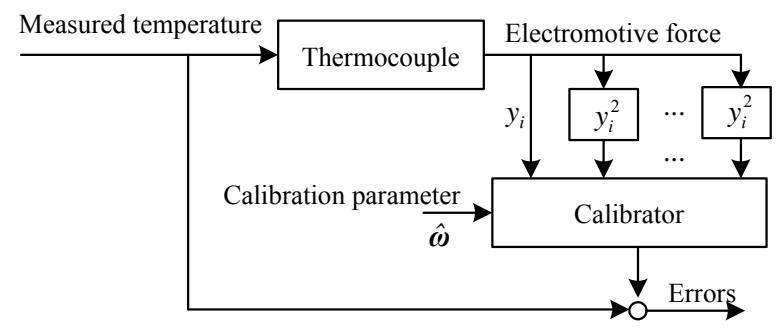

(b) Testing scheme of nonlinear calibrator

Fig .2 Calibration process of a thermocouple sensor using LS-SVR

\section{Identification of calibreation model}

According to formula (6), the indexing table of the platinum-rhodium 30 - platinum-rhodium 6 thermocouple (B-type) can be organized as training sample set $\left\{\mathbf{E}_{i}, \hat{x}_{i}\right\}_{i=1}^{M} \in R^{N} \times R$.

LS-SVR is a tool based on statistical learning theory, which can better deal with the relationship of empirical risk and expected risk under finite samples. To get better extensive ability, $\boldsymbol{\omega}^{T} \boldsymbol{\omega} / 2$ is led in the object, and the following optimization problem is formulated.

$$
\begin{aligned}
& \min \frac{1}{2} \hat{\boldsymbol{\omega}}^{T} \hat{\boldsymbol{\omega}}+\frac{1}{2} \gamma \sum_{i=1}^{M} e_{i}^{2}, \\
& \text { s.t. } \hat{x}_{i}=\hat{\boldsymbol{\omega}}^{T} \mathbf{E}_{i}+\hat{b}+e_{i}, i=1,2, \ldots, M,
\end{aligned}
$$


where the parameter $\gamma>0$. It is the regulating constant, which can get a proper to compromise between empirical risk and expected risk to make the generation ability of the undetermined function better. The larger the $\gamma$ is, the smaller the regression error of the model is.

In terms of the object function and constrained conditions in formula, vector $\hat{\boldsymbol{\omega}}$ can be solved in the dual space and the Lagrangian is given by

$$
L\left(\hat{\boldsymbol{\omega}}, \hat{b}, e_{i}, a_{i}\right)=\frac{1}{2} \hat{\boldsymbol{\omega}}^{T} \hat{\boldsymbol{\omega}}+\frac{\gamma}{2} \sum_{i=1}^{M} e_{i}^{2}-\sum_{i=1}^{M} a_{i}\left[\hat{\boldsymbol{\omega}}^{T} \mathbf{E}_{i}+\hat{b}+e_{i}-\hat{x}_{i}\right]
$$

with Lagrange multiplier $a_{i}, i=1,2, \ldots, M$. Thus, optimized $\mathbf{a}=\left[a_{1}, \ldots, a_{M}\right]^{T}$ can be solved on KKT condition as

$$
\begin{aligned}
& \frac{\partial L}{\partial \hat{\boldsymbol{\omega}}}=0 \rightarrow \hat{\boldsymbol{\omega}}=\sum_{i=1}^{M} a_{i} \mathbf{E}_{i}, \\
& \frac{\partial L}{\partial \hat{b}}=0 \rightarrow \sum_{i=1}^{M} a_{i}=0, \\
& \frac{\partial L}{\partial e_{i}}=0 \rightarrow \alpha_{i}=\gamma e_{i} \\
& \frac{\partial L}{\partial a_{i}}=0 \rightarrow \hat{\boldsymbol{\omega}}^{T} \mathbf{E}_{i}+\hat{b}+e_{i}-\hat{x}_{i}=0 .
\end{aligned}
$$

The optimization problem can be transformed to solve the matrix equation as follows

$$
\left[\begin{array}{l}
b \\
\boldsymbol{\alpha}
\end{array}\right]=\left[\begin{array}{cc}
0 & \overrightarrow{\mathbf{1}}^{T} \\
\overrightarrow{\mathbf{1}} & \boldsymbol{\Omega}+\gamma^{-1} \mathbf{I}
\end{array}\right]^{-1} \cdot\left[\begin{array}{c}
0 \\
\mathbf{U}
\end{array}\right],
$$

where $\boldsymbol{I}$ is the identity matrix, $\overrightarrow{\mathbf{1}}=[1, \ldots, 1]^{T}, \mathbf{X}=\left[\hat{x}_{1}, \ldots, \hat{X}_{N}\right]^{T}, \boldsymbol{\Omega}$ is a $N \times N$ square matrix, and

$$
\boldsymbol{\Omega}=\left[\begin{array}{cccc}
\mathbf{E}_{1}^{T} \mathbf{E}_{1} & \mathbf{E}_{1}^{T} \mathbf{E}_{2} & \ldots & \mathbf{E}_{1}^{T} \mathbf{E}_{N} \\
\mathbf{E}_{2}^{T} \mathbf{E}_{1} & \mathbf{E}_{2}^{T} \mathbf{E}_{2} & \ldots & \mathbf{E}_{2}^{T} \mathbf{E}_{N} \\
\ldots & \ldots & \ldots & \ldots \\
\mathbf{E}_{N}^{T} \mathbf{E}_{1} & \mathbf{E}_{N}^{T} \mathbf{E}_{2} & \ldots & \mathbf{E}_{N}^{T} \mathbf{E}_{N}
\end{array}\right]^{T}
$$

The vector a and $\hat{b}$ are obtained by solving equation (14), then the parameter $\hat{\boldsymbol{\omega}}$ of the modification inverse model can be estimated by substituting a into the formula.

$$
\hat{\boldsymbol{\omega}}=\sum_{i=1}^{N} a_{i} \mathbf{E}_{i}
$$




\section{Experiments}

The input-output characteristic of platinum-rhodium 30-platinum-rhodium 6 thermocouple (B-type) in the range of $0 \sim 1820^{\circ} \mathrm{C}$ is shown in Figure 3. Obviously, there is severe nonlinear in low-temperature, which has a direct impact on measurement accuracy [9].

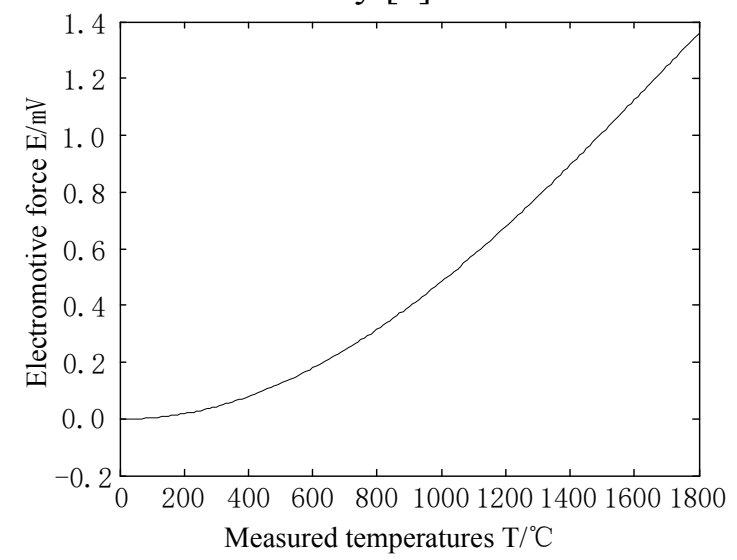

Fig.3 Input-output characteristics of platinum-rhodium 30-platinum-rhodium 6 thermocouple

Input-output characteristics of platinum-rhodium 30- platinum-rhodium 6 thermocouple Firstly, compose the training sample set $\left\{\boldsymbol{E}_{i}, x_{i}\right\}_{i=1}^{181} \in R^{N} \times R$ from the indexing table shown as Table 1. And then, identify the parameter $\hat{\omega}$ of the calibration model using LS-SVR, with the adjustment constan $\gamma=1000 \mathrm{t}$.

The order $N$ of power series polynomial is generally determined by the complexity of thermocouple's transfer characteristic curve. In this paper, when $N \geq 10$, the calibrator can achieve the better precision requirement. Finally, through the parameter estimation $\hat{\boldsymbol{\omega}}$, the calibration model was established as follows [9].

$$
\begin{aligned}
\hat{x}= & 1.123685 \times 10^{3} y-1.228351 \times 10^{3} y^{2}+8.157783 \times 10^{2} y^{3} \\
& -3.135332 \times 10^{2} E^{4}+7.388571 \times 10^{1} E^{5}-1.102232 \times 10^{1} E^{6} \\
& +1.044269 E^{7}-6.08787 \times 10^{-2} E^{8}+1.991155 \times 10^{-3} E^{9} \\
& +2.795738 \times 10^{-5} E^{10}
\end{aligned}
$$

The calibration process contains a platinum and rhodium 30 - platinum and rhodium 6 thermocouple and a nonlinear compensation model in a cascade. After calibration, the thermocouple's linearity can drop down to 0.0353 from 0.2123 , and the input-output characteristic of the whole system is shown in Figure 4.

we can see that the sub-function of the characteristics is taken on U-distribution in the range $0 \sim 100^{\circ} \mathrm{C}$. For example: for the same output electromotive force, the measured temperature may be $0^{\circ} \mathrm{C}$ and also $40^{\circ} \mathrm{C}$. Therefore, in the range of low-temperature, the inverse of the transfer function does not exist. On the other hand, in the high-temperature rang $\left(400 \sim 1800^{\circ} \mathrm{C}\right)$, the input-output characteristic is obviously monotonic, therefore the considerable desired calibration can be get with the inverse model. 


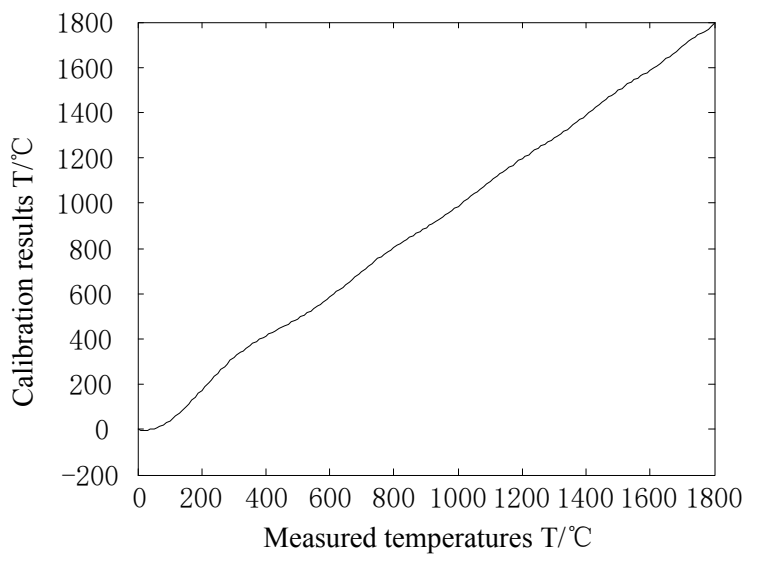

\section{Conclusions}

Fig.4 Input-output characteristic after calibraiton

By constructing the inverse model of transfer function, we can realize the nonlinear calibration of a sensor and improve the accuracy.

The inverse model was firstly expressed as power series polynomial, and LS-SVR was used to identify the model parameters. Difference from the conventional artificial intelligence methods using ANN, the proposed calibration method was a combination of modern technology (artificial intelligence) and traditional method (least-squares). The analytical expression of the calibration model can be obtained using the proposed method. Finally, the real examples of the platinum and rhodium 30platinum and rhodium 6 thermocouple (B-type) verified the feasibility of the proposed method.

\section{References}

[1] L.X. Tang, and P. Yan, "Particle swarm optimization algorithm for a batching problem in the process industry," Industrial and Engineering Chemistry Research, vol. 48, no. 20, pp. 9186-9194, October 21, 2009.

[2] Liu gang, "Data processing of intelligent sensor based on neural network," Transducer and Microsystem Technologies, vol. 23, pp. 52-54, August 2004.

[3] Huang tian-mao, and Ni Xiao-yong, "Study on nonlinearty correction of sensors by neutral network," Transducer and Microsystem Technologies, vol. 22, pp. 48-50, December 2003.

[4] N.V. Vladimir N, "An overview of statistical learning theory," IEEE Transactions on Neural Networks, vol. 10, no. 5, pp. 988-999, 1999.

[5] A.K. ohan, and J.V. Suykens, "Least squares support vector machine classifier," Neural Processing Letters, vol. 9, no. 3, pp. 293-300, 1999.

[6] R.Z. Morawski, "Unified approach to measurement signal reconstruction," Measurement, vol. 9, no. 3, pp. 141-144, 1991.

[7] J. Jakubiec, P. Makowski, and J. Roj, "Error model application in neural reconstruction of nonlinear sensor input signal," IEEE Transactions on Instrumentation and Measurement, vol. 58, no. 3, pp. 649656, 2009.

[8] J.C. Patra, G. Panda, and R. Baliarsingh, "Artificial neural network-based nonlinearity estimation of pressure sensors," IEEE Transactions on Instrumentation and Measurement, vol. 43, no. 6, pp. 874-881, Dec 1994.

[9] D.H. Wu, "Study on non-linearity compensation of temperature sensor based on LS-SVM," Transducer and Microsystem Technology, vol. 26, no. 9, pp. 35-37. 